\title{
Editorial
}

\section{Music Education in 2017}

One of the fascinating things about the articles in the BJME is that they come from many different countries, with many different music education systems in place in them. Each country has its own way of doing things, and its own way of thinking about what music education means in its own circumstance. One of the issues we face today is that with increasing globalisation unique ways of musicking can be under threat from nonindigenous commercial interests. For music education this can have implications. One of the questions that it raises is about the purposes of music education. Is music education about passing on a historic culture whose existence is threatened? Is it about learning to play western classical instruments? Is it about learning to play pop and rock music? These are not simple questions, and they do not have simple answers.

In the pages of BJME we meet articles which have their origins in music education systems which may be very different from our own. Reading them can cause us to ask ourselves why things are the way they are in our own context. But what happens when we work across contexts? Things can be very different even within the same jurisdiction. Thinking about what we have to do can raise interesting issues about style, genre and technique - as well as knowing and doing in music.

All of these things mean that to be a teacher of music today is a position which can involve a huge range of competences for one person, but very few for another. The matter of context is of crucial importance, and we need to consider it carefully before we venture too far down the road of thinking about music education.

Which brings us to this issue. We begin with a consideration of the role of emotional skills in music education, by Emilia-Ángeles Campayo-Muñoz and Alberto Cabedo-Mas from Spain. As they observe in their article, 'The link between music and emotion has therefore had an impact on educational activity, especially on teaching and learning music.' This in itself adds a further layer to the discussions of music education above; after all, music is a subject where there is clear evidence of emotional engagement, which may not always be the case in other subjects. For our next article, by Carolina Bonastre, Enrique Muñoz and Renee Timmers, we move to a not-unrelated construct: conceptions about teaching and learning of expressivity in music among Higher Education teachers and students, and we add a little bit of Sheffield in England - Yorkshire, to be precise - to the Spanish flavour we have had so far. In the following article, for which we move across to Portugal, Ana Luísa Veloso considers the role that collaborative creativity plays in developing children's composing. Again, this is an interesting perspective, as we know that in some countries composing plays only a small role in music education, so studies such as this, involving children in a Portuguese primary school, would be highly unusual for some.

Next, Maria Varvarigou considers for us the issue of 'playing by ear'. This is another example of a musical practice which does not figure consistently in music education curricula, yet which is vital in some forms. In this article, the classical musicians are described getting to grips with this powerful way of knowing music. Then we move around 
the globe, to see Vicki Thorpe use activity theory to analyse assessment of group composing for New Zealand's secondary school qualification. This clearly relates to other papers in this issue, and adds a useful analytical stratagem for close investigation of what takes place. For our final article in this issue, Christopher Dalladay, from England, describes what happens in generalist classroom learning in schools, another situation which is very much context-specific.

As you can see, we have again travelled far in our thinking about music education. As music education researchers and practitioners it behoves us to think about our own customs and practices in the light of what happens elsewhere. We are, after all, global citizens ourselves, and our learners will be making music that we know, and that we don't - yet.

MARTIN FAUTLEY

BJME Co-Editor 